\author{
New Method for Separation of Skeletal Material from a Plankton Sample* \\ GUSTAVS VILKS \\ Atlantic Oceanographic Laboratory, Bedford Institute, Dartmouth, N. S.
}

$\underline{\text { Introduction }}$

In a plankton sample the presence of small-shelled animals such as foraminifera, radiolaria, etc., is often obscured by larger organisms. For systematic work on foraminifera an efficient method of extraction that fulfills at least the following three requirements is necessary: 1) accuracy and speed, 2) preservation of the fine morphological structures of the tests, and 3) the removal of all organic matter.

All the most widely known methods described in the literature fall short of these requirements. In the "classical" method described by Be (1969a) and Boltovskoy (1966), the total . sample is inspected through a microscope and any foraminiferal tests visible are removed by a pipette or a brush. The method is time-consuming and inaccurate in that the worker is unconsciously selective towards larger and more conspicuous species such as the red Globigerinoides miber or Hastigerina pelagica. Another source of biased error is the element of fatigue, which causes a decrease in operator accuracy through the working period.

In the floatation method proposed by $\mathrm{Be}$ (1959b), a saturated $\mathrm{NaCl}$ solution is used to separate shelled from soft-bodied plankton. When placed in this solution in a settling tube, ideally the soft-bodied plankton remain floating while foraminifera, with the other shelled organisms, sink to the bottom. A major draw-back of this method is that spinose species are commonly entangled with the other soft-bodied plankton and remain floating.

By the ignition method, proposed by Sachs et al (1964) samples are initially treated with hydrogen peroxide. After the excessive soft organic material is digested, the samples are ignited in a muffle furnace at $500^{\circ} \mathrm{C}$. This treatment removes all organic material, leaving only skeletal matter. Both the initial treatment with the digestive and the final treatment in the furnace attacks the calcareous material to some extent. For example, the surfaces of the tests of foraminifera and pteropods is etched and most of the spines are removed in this process. In many instances it was found that samples had exploded in the furnace causing loss and contamination of the sample.

\title{
Description of New Method
}

The method proposed here consists of oxidizing the organic matter of a plankton sample by the use of the TRACERLAB LTA-600** low-temperature asher with five ashing chambers. The sample is exposed to a flow of oxygen which is excited by a radio-frequency electromagnetic field. In this state the oxygen readily reacts with organic matter and, because this is the only means of energy input, organic substances are removed from the sample at temperatures not exceeding $150^{\circ} \mathrm{C}$. The reaction takes place in a vacuum between $0.1-1.0 \mathrm{~mm}$ of $\mathrm{Hg}$. For the study of planktonic foraminifera the following working procedure was adopted. Wash either the whole sample or an aliquot with distilled water through a 60-micron sieve to remove preservative solution. Transfer the sample to a Millipore filter by using a stream of distilled water. Filter the sample and dry for at least four hours at $60^{\circ} \mathrm{C}$ on the Millipore filter-pad. Transfer the dried sample with the filter-pad on a TRACERLAB sample boat and ash the sample. A dried sample weighing $0.1-0.2$ grams will oxidize (ash) completely in about seven hours. The membrane filter oxidizes readily, leaving a negligible amount of ash. When oxidation is complete, carefully remove the sample boat from the asher and wash the concentrate again through the 60-micron sieve using a mild stream of distilled water to remove all ash. Wash the clean sample in a Millipore filtering apparatus using a gridded black filter. Place the filter-pad with the sample in a disposable Millipore petri-dish, the bottom of which is covered with glue. Dry the sample and close the dish.

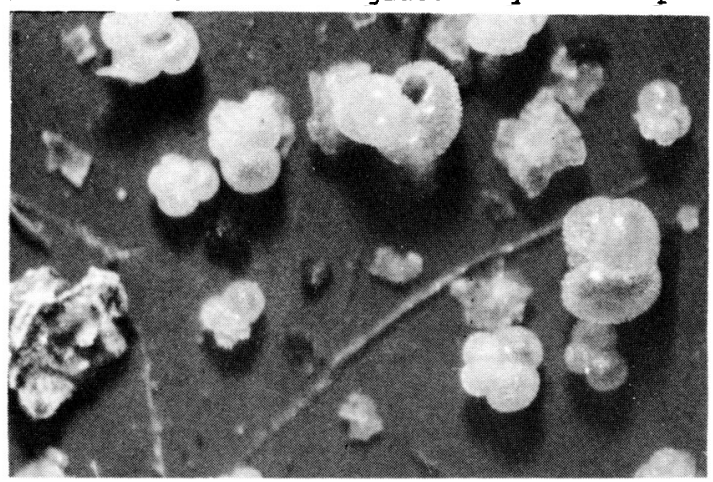

Figure 1 - Ashed sample with the black Millipore filter as background. Sample 174B, Magnification approximately 100 times.

* Manuscript received July 29, 1970.

** TRACERLAB, 2030 Wright Ave., Richmond, California. 
Discussion of the Method

The process of oxidation in the TRACERLAB asher is so mild that the calcareous or siliceous material in the sample is completely unharmed (Fig. 1). Organic material however is removed completely, allowing easy access to the skeletal matter of the sample. After a run is started the instrument does not require any attention during the operation. By transferring the sample with a stream of water only, loss of material is minimized. The ashed sample is stored in the petridish where it can also be viewed with the microscope. The gridded black filter provides an excellent background for counting and studying morphological features of the test. This is desirable because it minimizes the time and effort required for faunal analysis. Specimens intended for study under the scanning electron microscope are sufficiently clean and do not require any additional treatment.

To test the efficiency of the new method, 10 plankton samples were split into four parts and treated by four different methods (See Table I). The values of Table I indicate that the ashing method is the most efficient of the four methods tested. Except for sample 174A, the fraction of samples that were treated with the low temperature asher produced the largest numbers of foraminifera. The larger counts are probably due to the preservation of juvenile or other fragile tests. In the furnace method these tests are usually broken or destroyed, but in the "classical" method the small and transparent juveniles are difficult to see and could easily be missed. In the method that uses the settling tube, the small tests are not separated from the remainder of the plankton because of the similarity of the buoyancies.

Table I. Number of planktonic foraminifera counted in each quarter of a sample using four different methods of sample preparation.

LOCATION COUNTS OF FORAMINIFERA FOR EACH METHOD

\begin{tabular}{|c|c|c|c|c|c|c|c|}
\hline $\begin{array}{l}\text { Sample } \\
\text { No. }\end{array}$ & Lat.N. & Long.W. & $\begin{array}{l}\text { Date } \\
1968\end{array}$ & Asher & Furnace & Float & "Classical" \\
\hline 155 & $45^{\circ} 51^{\prime}$ & $29^{\circ} 53^{\prime}$ & $28 / 8$ & 95 & lost & 54 & 58 \\
\hline 157 & $45^{\circ} 49^{\prime}$ & $29^{\circ} 09^{\prime}$ & " & 161 & 48 & 14 & 101 \\
\hline $161 \mathrm{~A}$ & $45^{\circ} 40^{\prime}$ & $29^{\circ} 19^{\prime}$ & $"$ & 132 & 71 & 33 & 49 \\
\hline $161 \mathrm{~B}$ & $"$ & $"$ & $"$ & 64 & 60 & 41 & 25 \\
\hline $161 \mathrm{C}$ & $"$ & $"$ & $"$ & 139 & 18 & 53 & 65 \\
\hline 164 & $45^{\circ} 14^{\prime}$ & $29^{\circ} 52^{\prime}$ & $29 / 8$ & 121 & 65 & 16 & 48 \\
\hline $167 \mathrm{~A}$ & $45^{\circ} 16^{\prime}$ & $27^{\circ} 59^{\prime}$ & $30 / 8$ & 35 & 3 & 16 & 20 \\
\hline $167 \mathrm{~B}$ & $"$ & $"$ & $"$ & 111 & 8 & 50 & 73 \\
\hline $174 \mathrm{~A}$ & $45^{\circ} 42^{\prime}$ & $27^{\circ} 47^{\prime}$ & $31 / 8$ & 106 & 126 & 65 & 103 \\
\hline $174 \mathrm{~B}$ & $"$ & $"$ & $"$ & 394 & 390 & 112 & 194 \\
\hline Mean & & & & 135 & 79 & 45 . & 74 \\
\hline
\end{tabular}

The laboratory procedure proposed here has several other advantages in terms of improved accuracy and speed. Sample loss is minimized by advoiding the transfer of dry samples, thus allowing extremely small samples to be handled without introducing significant errors. The complete removal of all organic matter and the storage of the clean sample on the black membrane filter minimizes the effort of identifying and counting the foraminifera.

\section{References cited}

BE, A.W.H, 1959a, Ecology of Recent planktonic foraminifera: Part I - Areal distribution in the western North Atlantic. Micropaleontology, 5, 77-100.

, 1959b, A method for rapid sorting of foraminifera from marine plankton samples. J. Paleont., 33, 846-848.

BOLTOVSKOY, E., 1966, Method for sorting of foraminifera from plankton samples. J. Paleont., 40, $1244-1246$.

SACHS, K.N., Jr., CIFELLI, R., and BOWEN, T., 1964, Ignition to concentrate shelled organisms in plankton samples. Deep-Sea Res., 11, 621-622. 